\title{
Epstein-Barr Virus-Negative Hodgkin's Lymphoma After Mycosis Fungoides: Molecular Evidence for Distinct Clonal Origin
}

\author{
Marcus Kremer, M.D., Michael Sandherr, M.D., Birgit Geist, Antonello D. Cabras, M.D., \\ Heinz Höfler, M.D., Falko Fend, M.D. \\ Institute of Pathology ( $M K, B G, A D C, H H, F F$ ), Technical University Munich, Germany; Department of \\ Internal Medicine III (MS), Technical University Munich, Germany; and Institute of Pathology (MK), \\ GSF-National Research Center for Environment and Health, Neuherberg, Germany
}

The association of mycosis fungoides (MF) and Hodgkin's lymphoma is a relatively frequent occurrence, but the potential clonal relationship of the two neoplasms is still controversial. We report a case of a patient with a history of MF in Clinical Stage $1 \mathrm{~A}$ who developed retroperitoneal lymphadenopathy 9 years after the initial diagnosis of MF. A bone marrow biopsy obtained at this time showed nodular involvement by a mixed cellular infiltrate with large, atypical cells consistent with Hodgkin and Reed-Sternberg (RS) cells. These atypical cells were positive for CD30 and CD15 and did not express B- or T-cell markers. In addition, they lacked evidence of infection by Epstein-Barr virus, both by immunohistochemical staining for latent membrane protein 1 and by in situ hybridization for EBER1/2. The background population consisted mainly of small $\mathrm{T}$ cells without morphological or phenotypical signs of malignancy. Review of the skin biopsy obtained 9 years before showed the typical features of MF. Polymerase chain reaction analysis of the T-cell receptor $\gamma$-gene confirmed the presence of a clonal $T$-cell rearrangement in the skin specimen. The bone marrow biopsy, however, showed a polyclonal pattern both for the T-cell receptor $\gamma$-gene, as well as for immunoglobulin heavy chain genes. Isolation of RS cells stained for CD30 was performed by laser capture microdissection. Polymerase chain reaction analysis of several groups of RS cells showed a reproducible biallelic rearrangement of IgH genes, which was confirmed by cloning and sequencing of polymerase chain reaction products. To our knowledge, this is the first

Copyright () 2001 by The United States and Canadian Academy of Pathology, Inc.

VOL. 14, NO. 2, P. 91, 2001 Printed in the U.S.A.

Date of acceptance: September 25, 2000.

Address reprint requests to: Fend Falko, M.D., Institute of Pathology,

Technical University Munich, Ismaningerstrasse 22, D-81675 Munich,

Germany; email: Fend@Irz.tum.de; fax: 49-89-4140-6106. case in which a distinct clonal origin of MF and Hodgkin's lymphoma arising in the same patient is clearly demonstrated, based on molecular analysis of microdissected RS cells.

KEY WORDS: Bone marrow, Epstein-Barr virus, Microdissection, Hodgkin's lymphoma, Mycosis fungoides, PCR, TCR $\gamma$, IgH.

Mod Pathol 2001;14(2):91-97

The association of Hodgkin's lymphoma (HL) and non-Hodgkin's lymphoma (NHL) in the same patient has been reported repeatedly and can present in a variety of settings. The two neoplasms can occur simultaneously, or the diagnosis of HL can precede or follow a diagnosis of NHL (1). Among peripheral T-NHL, mycosis fungoides (MF) is the neoplasm most commonly associated with HL (28). The relatively high frequency of this association has led some authors to speculate that MF and HL are derived from the same clone. Davis et al. (3) demonstrated a common single $\mathrm{T}$-cell clone for lymphomatoid papulosis, MF, and HL occurring in the same patient. In contrast, other authors have not found evidence for a common clonal origin of MF associated with HL $(2,4)$. A distinct phenomenon potentially related to the association of HL and NHL is the presence of cells morphologically and, in part, also phenotypically indistinguishable from typical Hodgkin and Reed-Sternberg (RS) cells in tissues involved by NHL of both B- or T-cell origin $(9-14)$. Because of the technical obstacles for molecular analysis, only very few studies have tried to investigate the clonal relationship between HL and NHL occurring in the same patient or the origin of RS-like cells present in NHL.

Recently, the advent of microdissection and single-cell polymerase chain reaction (PCR) analysis allowed the determination of the lineage and clonality of HL and established a B-cell genotype in 
most instances $(15,16)$. This methodical approach has also been applied to some cases of HL and NHL of B-cell phenotype arising in the same patient. Using microdissection of individual tumor cells, a common clonal origin of the two neoplastic populations has been demonstrated in most but not all of these cases (17-19).

To our knowledge, a molecular analysis of isolated RS cells has not been performed so far in cases of HL associated with cutaneous T-NHL. We used laser capture microdissection of RS cells to investigate the clonality and lineage of a HL arising in a patient with a history of MF.

\section{Case Report}

A 50-year-old male patient with an unremarkable medical history presented with multiple cutaneous plaques and nodules two years prior to the diagnosis of MF on a skin biopsy in 1989. After clinical staging, the disease was classified as Stage 1A. The patient received several photochemotherapies with UVA radiation and reached complete remission of the skin lesions. Seven years later, cutaneous plaques reappeared and diagnosis of recurrent MF was rendered on a skin biopsy. A bone marrow (BM) biopsy obtained at the same time showed small nodular lymphoid infiltrates, which were interpreted as involvement by MF. One year later, in 1997, hepatosplenomegaly and retroperitoneal lymphadenopathy developed and were interpreted as generalization of the cutaneous T-cell NHL. Polychemotherapy with mitoxantrone, chlorambucil, and prednisolone was initiated. In 1998, pancytopenia developed. A repeat bone marrow biopsy revealed a marked nodular lymphoid infiltrate with interspersed Hodgkin and Reed-Sternberg cells. A diagnosis of an aggressive transformation of MF with HL-like features was made. The patient was treated with Endoxan and died 4 months later. An autopsy was not performed.

\section{MATERIALS AND METHODS}

A formalin-fixed, paraffin-embedded tissue block from the initial skin biopsy (1989) as well as buffered ( $\mathrm{pH}$ 7.4) formalin- (1\%)/glutaraldehyde (0.5\%)-fixed and buffered Na-EDTA (pH 7.0) decalcified (48 h), paraffin-embedded paraffin tissue blocks from the BM biopsies $(1996,1998)$ were available. Fourmicrometer-thick sections were cut and were stained with hematoxylin and eosin (H\&E; skin biopsy and BM biopsy), Giemsa, Gomori's reticulin, and Naphtol AS-D chloroacetate esterase (BM).

\section{Immunophenotyping and In Situ Hybridization}

Immunohistochemical studies were performed on paraffin sections using an automated immunos- tainer (DAKO, Hamburg, Germany) according to the company's protocol with minor modifications. The antibody panel used included CD20 (L26), CD3 (polyclonal), CD45RO (UCHL-1), CD4 (MT310), CD8 (DK25), CD30 (Ber-H2; all from DAKO Diagnostika, Hamburg, Germany), CD43 (Leu22), and CD15 (Leu M1; those last two from Becton Dickinson, San Jose, CA). The BM biopsy was evaluated for the presence of Epstein-Barr virus (EBV) latent membrane protein 1 (LMP-1, CS 1-4, DAKO).

In situ hybridization for EBV early RNAl/2 (EBERs) was performed on sections of the skin and the BM biopsies (1996, 1998; PNA ISH Detection Kit, DAKO) according to the manufacturer's instructions. A case of EBV-positive HL was used as a positive control.

\section{Laser Capture Microdissection}

Laser capture microdissection (LCM) was performed on paraffin sections of the BM biopsy from 1998 stained for CD30, using a PixCell II laser capture microscope with an infrared diode laser and a spot size of $7.5 \mu \mathrm{m}$, allowing single-cell microdissection (Acturus Engineering, Santa Clara, CA) as previously described $(12,20)$. Four different microdissections were performed separately, collecting approximately 15 RS cells each, pooled on a dissection membrane. Each cell group was digested with proteinase $\mathrm{K}$ in a total volume of $20 \mu \mathrm{L}$. As polyclonal control, 50 to 60 cells were randomly picked from germinal centers of a lymph node with chronic lymphadenitis.

\section{Molecular Genetic Studies}

Genomic DNA was extracted from the skin biopsy and both bone marrow biopsies and used to assay for clonal rearrangements of the immunoglobulin heavy chain $(\mathrm{IgH})$ genes and the T-cell receptor (TCR) $\gamma$ genes by PCR, using published protocols $(21,22)$. For the microdissected cells, a seminested PCR procedure was employed for amplification of the complementary-determining Region III of the IgH gene employing primers directed against a consensus sequence of the third-frame work region (Fr3a) of the variable regions and consensus sequences of the joining regions (LJH, VLJH), as described elsewhere, with minor modifications (12). Briefly, primer pairs FR3A-LJH (5'-ACA CGG CCG TGT ATT ACT GT, 5'-TGA GGA GAC GGT GAC C) and FR3A-VLJH (5'-GTG ACC AGG GTX CCT TGG CCC CAG) were used for 40 and 25 cycles of amplification, respectively. Six microliters of the crude extract served as template for the first round of amplification, and $5 \mu \mathrm{L}$ of the undiluted external PCR product was used for the second round using the same temperature profile. PCR reactions were 
repeated to ensure reproducibility. Negative controls included water, DNA extraction buffer, and unused LCM caps. PCR reaction products were electrophoresed in a 3\% Metaphor gel. In addition, the first-round product was reamplified with a fluorescence-labeled VLJH primer for analysis with a computer-assisted fragment length analysis system using a high-resolution polyacrylamide gel for size determination (Genescan, Perkin Elmer, Weiterstadt, Germany; 23).

\section{Cloning and Sequencing of PCR Products}

Clonal amplification products were extracted from agarose gel slices using the QIAquick gel extraction Kit (Qiagen, Hilden, Germany). The PCR products were cloned with the PCR-Script Amp Cloning kit (Stratagene, Amsterdam, The Netherlands). A minimum of five clones was sequenced using the BigDye Terminator cycle sequencing kit (Perkin Elmer, Weiterstetten, Germany) and an ABI Prism 377 automated sequencer.
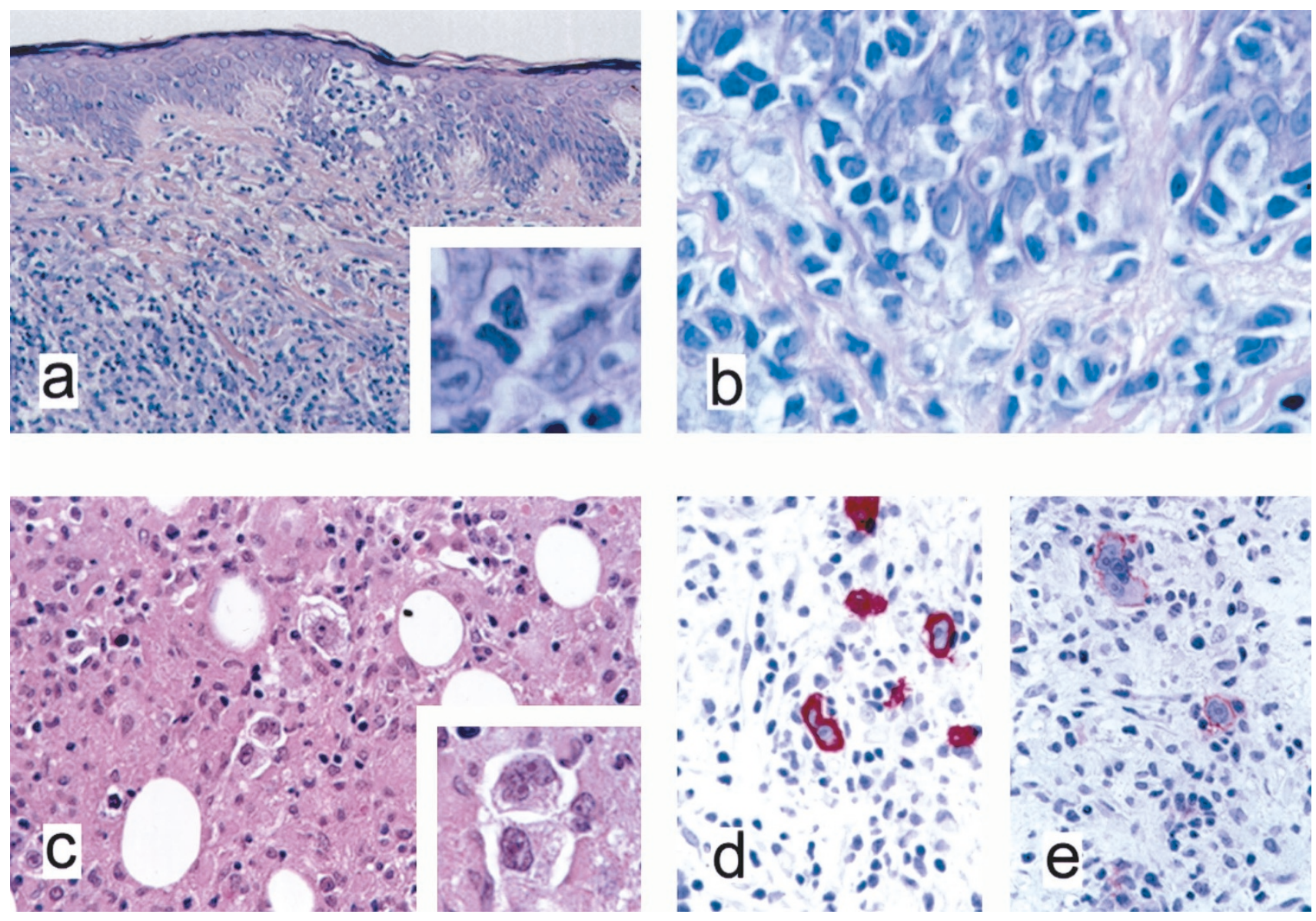

\section{RESULTS}

\section{Pathologic Findings}

The cutaneous lesions from 1989 showed the typical histologic features of MF. The biopsy contained band-like, dermal, sometimes-perivascular and focally epidermotropic lymphoid infiltrates containing atypical lymphocytes with hyperchromatic and irregularly shaped nuclei, admixed with scattered blasts and inflammatory eosinophils and plasma cells. The epidermis contained single or small clusters of atypical lymphocytes with cerebriform outlines (Pautrier microabscess). Some of the lymphocytes showed a clear, perinuclear halo. (Fig. 1a-b) The BM biopsies from 1996 and 1998 showed lymphoid infiltrates characterized by small lymphocytes with admixed eosinophils and plasma cells. The BM biopsy from 1998 revealed, in addition, scattered large mononuclear and binucleated cells with prominent eosinophilic nucleoli with abundant cytoplasm resembling RS cells and mononuclear variants. (Figure 1c)

FIGURE 1. Histopathologic and immunohistochemical features. (A) Mycosis fungoides: band-like subepidermal and focally epidermotropic lymphoid infiltrates. Note the characteristic intraepidermal cluster of lymphocytes (Pautrier microabscess); the inset shows atypical intraepidermal lymphocytes, H\&E staining, magnification $\times 1000$. (B) Microphotograph of the epidermal-subepidermal junction with intraepidermal lymphocytes and haloed lymphocytes in the basal layer, magnification $\times 600$. (C-E) Bone marrow: lymphoid infiltrate consisting of small lymphocytes with scattered large mononuclear and binucleated cells $(\mathbf{C})$; the inset shows typical Hodgkin and RS cells, magnification $\times 400$. The Hodgkin and RS cells show a strong membrane staining for (D) CD15 and (E) CD30. Magnification of Panels D and E: $\times 300$. 


\section{Immunophenotypic Findings}

The lymphoid infiltrates in the skin biopsy showed strong immunoreactivity for CD3, CD45R0, and CD43 and showed a predominance of CD4+ cells. Only rare CD20+ small lymphocytes were present. Occasional larger cells showed weak CD30 positivity but were negative for CD15.

The lymphoid infiltrates in the BM biopsy from 1996 revealed strong staining for T-cell markers (CD3, CD4, CD8, and CD45R0) and contained rare CD20-positive lymphocytes. The Hodgkin and RS cells of the BM biopsy (1998) showed strong membrane staining for CD15 and CD30. (Fig. 1d-e) They were negative for CD20, for all investigated T-cell markers (CD3, CD4, CD8, CD43, and CD45R0), and for the EBV latent membrane protein 1 (LMP-1), as well as negative for EBERs by in situ hybridization. Only rare B cells were identified by CD20 staining; the majority of the small lymphoid cells in the infiltrates stained for T-cell markers.

\section{Molecular Findings}

PCR of the TCR $\gamma$ was performed in all available specimens. The skin biopsy showed single clonal bands of 67 and 80 base pairs, respectively, with the two primer sets as revealed by fragment length analysis (data not shown). In contrast, TCR $\gamma$ PCR of the BM sections (1996 and 1998) resulted in a reproducible polyclonal pattern. Amplification of the pooled, microdissected Hodgkin and RS cells gave occasional, irreproducible oligoclonal bands, probably because of amplification of rare $\mathrm{T}$ cells adherent to the microdissected RS cells.

PCR assays for the IgH genes performed on the skin biopsy and whole sections of the bone marrow biopsies showed a polyclonal pattern. IgH PCR of three different groups of microdissected RS cells from the BM biopsy of 1998 resulted in amplification of identical clonal bands of 79 and 82 base pairs, respectively. All three PCR products were cloned. Two sequences of appropriate lengths were obtained, which were found in eight and seven clones, respectively, originating from the three different PCR products. The amplification of the same sequences from three different groups of RS cells confirmed the presence of a biallelic clonal IgH rearrangement. (Fig. 2c-e)

On the basis of the morphological, immunophenotypical and molecular findings, the bone marrow findings in 1998 were interpreted as a secondary, unrelated neoplasm consistent with classical HL.

\section{DISCUSSION}

The present study describes a case of HL arising in a patient with a long history of MF. The immunophenotypic findings and the molecular analysis of microdissected RS cells confirm that the HL in this patient represents a true secondary neoplasm of B-cell origin, clonally unrelated to the precedent cutaneous T-NHL.

The synchronous or metachronous occurrence of Hodgkin's lymphoma and T-cell NHL in the same patient has been reported repeatedly. The most commonly described association is that of MF and HL (3-8). MF usually appears before HL, but the reverse has been also documented (24). Only a minority of these cases have been investigated by molecular methods, and the findings concerning a clonal relatedness of the two neoplasms are controversial. Whereas a single study using clone-specific primers and probes demonstrated a common T-cell clone in a patient with HL, cutaneous T-cell NHL, and lymphomatoid papulosis (3), other authors have not confirmed these findings $(2,4)$. However, none of these studies have used microdissection to definitively determine the lineage and clonality of the neoplastic cells of the HL.

In HL, microdissection and single-cell analysis have proved to be crucial for resolving the longstanding controversy concerning the origin and clonality of the neoplastic cells. The vast majority of cases have been shown to be of germinal center B-cell origin, although a well-documented case of HL with T-cell phenotype has recently been reported $(15,16,25)$. The same technique has also been used to study the clonal relationship of HL and B-NHL arising in the same patient. A common clonal B-cell precursor has been identified in patients developing HL in the setting of B-CLL and in HL associated with follicular lymphoma and T-cellrich B-cell lymphoma, respectively $(18,19)$.

A possibly related phenomenon is the occurrence of RS-like cells in the background of NHL of T- or B-cell origin, which traditionally has been interpreted as transformation of the initial tumor cell clone $(6,9-11,13,14,26,27)$. In chronic lymphocytic leukemia of B-cell origin, these RS-like cells are usually EBV positive and frequently show the typical phenotype of RS cells of classical HL. Recent studies using microdissection techniques showed that RS-like cells and the neoplastic chronic lymphocytic leukemia cells are frequently, but not always, clonally related $(17,19)$.

RS-like large cells can also be observed in peripheral T-cell lymphoma, including aggressive transformation of MF. They are often part of a continuous spectrum of atypical cells and frequently retain expression of T-cell markers, suggesting that they are part of the malignant clone. In a patient with MF followed by CD30+ large-cell lymphoma, a common clonal origin has been documented by molecular studies (28).

A distinct phenomenon is the appearance of RSlike cells expressing B-cell markers in some periph- 

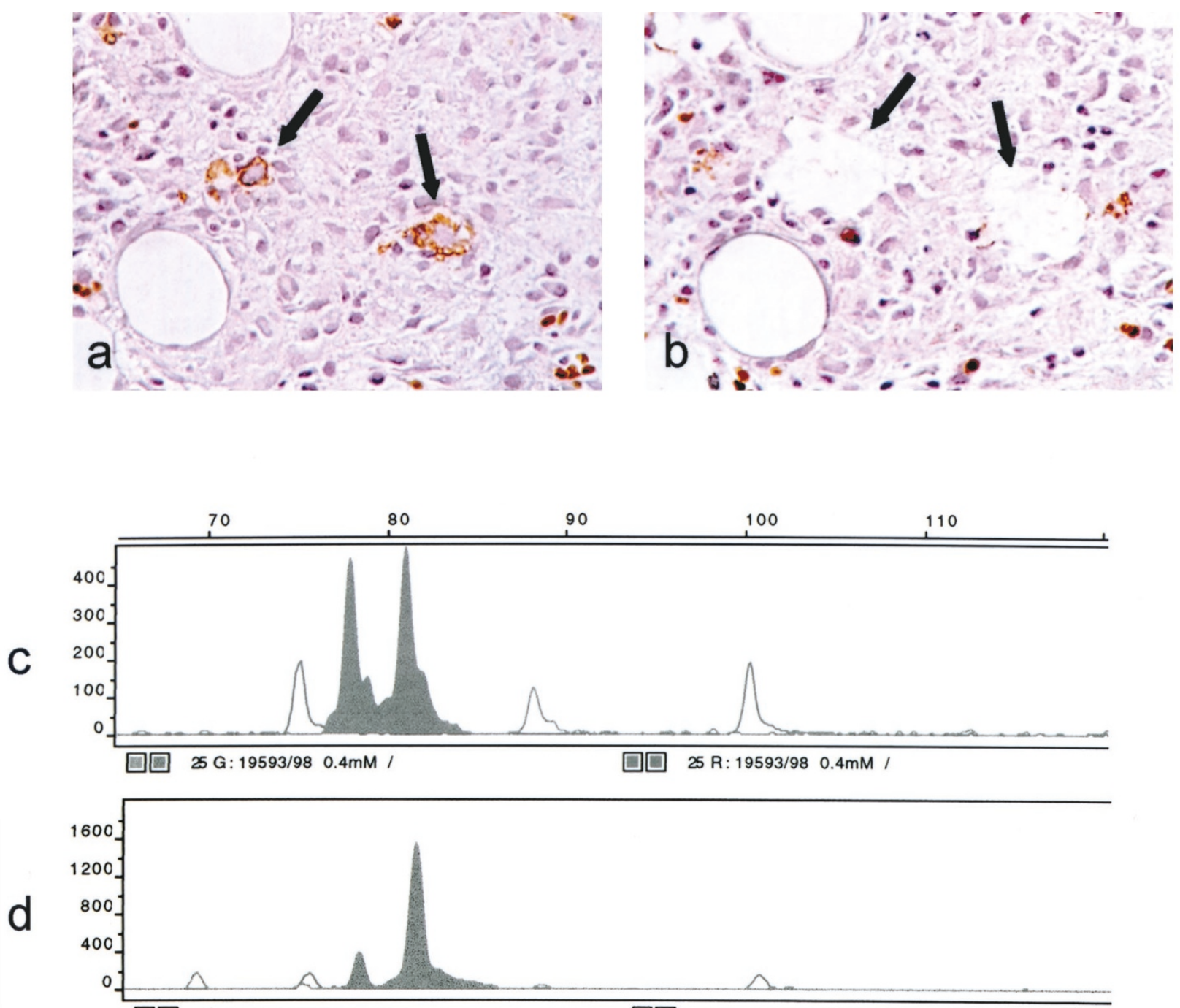

$\square$ 20 G: $19593 / 98$ 0.2mM / $\square \square$ 29 R: $19593 / 980.2 \mathrm{mM} /$

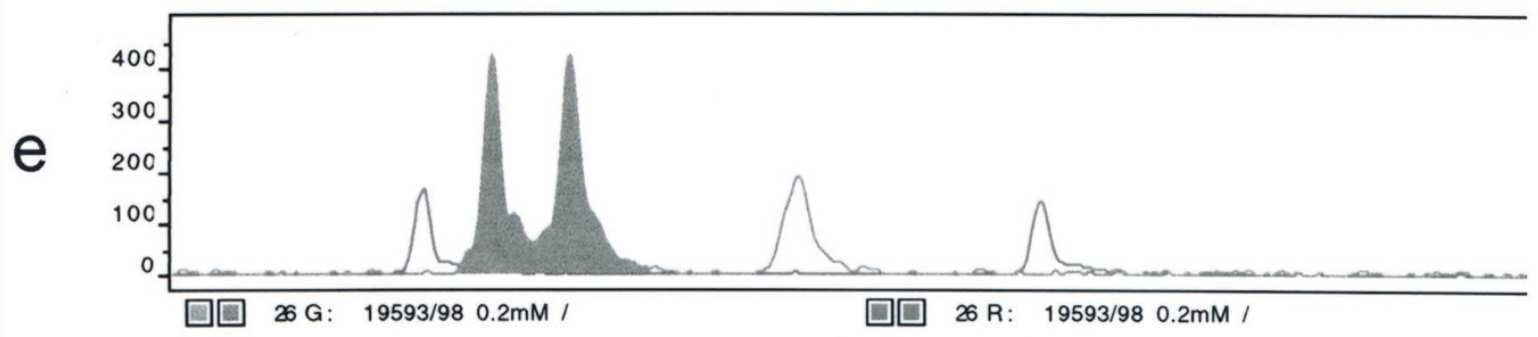

\section{$5{ }^{`}$-end $\mathrm{Fr} 3 / \mathrm{N}$ TGTGCGAGAATT GGGTATAGCAGCTCGTCC}

f

\section{5'-end $\mathrm{Fr} 3 / \mathrm{N}$ \\ D6-13 \\ TGTACTAGAGGA GGTATAGCAGCACCCT}

FIGURE 2. (A) Bone marrow infiltrate with small lymphoid cells and typical Hodgkin and RS-cells. The RS-cells were stained for CD30 (arrows). (B) the same CD30-stained area as in Panel A after LCM of CD30-positive RS cells (arrows). Note that the photograph is taken without cover slide in Panels A and B $(\times 400)$. (C-E) Computer-assisted fragment length analysis (Genescan) of three different groups of microdissected CD30-positive RS cells with identical biallelic rearrangements for the IgH gene. (F) Corresponding rearranged IgH gene sequences obtained from microdissected cells. 
eral T-cell lymphomas $(11,12,27)$. In incipient adult T-cell leukemia/lymphoma with Hodgkin-like features, Oshima et al. (29) demonstrated the presence of unrelated immunoglobulin gene rearrangements in isolated RS-like cells. Similarly, Quintanilla-Martinez et al. (12) found polyclonal immunoglobulin gene rearrangements in EBV-positive RS-like cells isolated by LCM from cases of peripheral T-cell lymphoma with angioimmunoblastic features. Thus, these cells seem to represent a polyclonal, EBV-transformed reactive population unrelated to the malignant clone. Defective T-cell function and underlying immunodeficiency may predispose to expansion of EBVtransformed B-cells in these NHLs $(12,29)$. Notably, none of these lymphomas showed evidence of progression to HL.

Our case differs from the two phenomena described above in several aspects. Although predominantly of T-cell phenotype, the background lymphocytes in the bone marrow infiltrate, in contrast to the skin lesion, lacked cellular atypia and a clonal $\mathrm{TCR} \gamma$ rearrangement and therefore represent a reactive infiltrate. Moreover, the RS cells showed a clonal rearrangement of the IgH gene, documented by amplification of identical IgH rearrangements from independent groups of isolated RS cells, which is indicative of a true secondary neoplasm. Even though no biopsy of the retroperitoneal lymphadenopathy was obtained, the morphologic and immunophenotypical findings on the bone marrow biopsy in conjunction with the molecular findings are consistent with a diagnosis of classical HL.

In contrast to the RS-like cells in other T- and B-cell lymphomas, these cells lacked EBV genome. The EBV negativity of our case is in accordance with other reports showing absence of EBV infection in the majority of cases of HL associated with MF (4, 30 ). The relatively frequent association of EBVnegative HL and MF could point to a so-farunidentified common etiologic agent or to shared risk factors. Zucker-Franklin et al. (31) reported the release of retroviral particles from cultured lymphocytes of $18 \mathrm{MF}$ patients. However, to date, there is no convincing evidence for an involvement of either human T-cell Lymphotropic Virus Type-I nor human Herpesvirus 8 in the pathogenesis of MF $(32,33)$. Patients with HL show defects in cellmediated immunity that can precede the development of the disease (34). Antecedent chemotherapy and MF-associated immunosuppression could probably enhance the defects in cell-mediated immunity and therefore development of subsequent HL.

In conclusion, our case provides evidence that EBV-negative HL arising in association with MF can represent a true secondary, clonally unrelated neoplasm of B-cell origin. This finding, based on molecular analysis of microdissected RS cells, confirms and extends previous reports using immunohistochemical and molecular techniques $(2,4,7)$. In the light of the existence of rare cases of HL with a $\mathrm{T}$-cell origin, further studies will be needed to exclude the possibility of rare cases of MF and HL with a common clonal T-cell origin. $(3,25)$.

Acknowledgments: The authors are grateful to Dr. Leticia Quintanilla-Martinez for critical reading of the manuscript and to Sabine Noll for help with the immunohistochemistry.

\section{REFERENCES}

1. Jaffe ES, Zarate-Osorno A, Medeiros LJ. The interrelationship of Hodgkin's disease and non-Hodgkin's lymphomas-lessons learned from composite and sequential malignancies. Semin Diagn Pathol 1992;9:297-303.

2. Geldenhuys L, Radhi J, Hull PR. Mycosis fungoides and cutaneous Hodgkin's disease in the same patient: a case report. J Cutan Pathol 1999;26:311-4.

3. Davis TH, Morton CC, Miller-Cassman R, Balk SP, Kadin ME. Hodgkin's disease, lymphomatoid papulosis, and cutaneous T-cell lymphoma derived from a common T-cell clone. N Engl J Med 1992;326:1115-22.

4. Brousset P, Lamant L, Viraben R, Chlaifer D, Gorguet D, Duhault $\mathrm{P}$, et al. Hodgkin's disease following mycosis fungoides: phenotypic and molecular evidence for different tumour cell clones. J Clin Pathol 1996;49:504-7.

5. Chan WC, Griem ML, Grozea PN, Freel RJ, Variakojis D. Mycosis fungoides and Hodgkin's disease occurring in the same patient: report of three cases. Cancer 1979;44:1408-13.

6. Salhany KE, Cousar JB, Greer JP, Casey TT, Fields JP, Collins RD. Transformation of cutaneous T-cell lymphoma to large cell lymphoma. A clinicopathologic and immunologic study. Am J Pathol 1988;132:265-77.

7. Simrell CR, Boccia RV, Longo DL, Jaffe ES. Coexisting Hodgkin's disease and mycosis fungoides. Immunohistochemical proof of its existence. Arch Pathol Lab Med 1986; 110:1029-34.

8. Caya JG, Choi H, Tieu TM, Wollenberg NJ, Almagro UA. Hodgkin's disease followed by mycosis fungoides in the same patient. Cancer 1984;53:463-7.

9. Momose H, Jaffe ES, Shin SS, Chen YY, Weiss LM. Chronic lymphocytic leukemia/small lymphocytic lymphoma with Reed-Sternberg-like cells and possible transformation to Hodgkin's disease. Mediation by Epstein-Barr virus. Am J Surg Pathol 1992;16:859-67.

10. Oshima K, Kikuchi M, Yoshida T, Masuda Y, Kimura N. Lymph nodes in incipient adult T-cell leukemia-lymphoma with Hodgkin's disease-like histologic features. Cancer 1991; 67:1622-8.

11. Ott G, Ott MM, Muller-Hermelink HK. Epstein-Barr virus, B-cells, T-cells, and a lymphoproliferative disorder [letter]. Lancet 1994;343:922.

12. Quintanilla-Martinez L, Fend F, Rodriguez Moguel L, Spilove L, Beaty M, Kingma D, et al. Peripheral T-cell lymphoma with Reed-Sternberg-like cells of B-cell phenotype and genotype associated with Epstein-Barr Virus infection. Am J Surg Pathol 1999;23:1233-40.

13. Rubin D, Hudnall SD, Aisenberg A, Jacobson JO, Harris NL. Richter's transformation of chronic lymphocytic leukemia with Hodgkin's-like cells is associated with Epstein-Barr virus infection. Mod Pathol 1994;7:91-8.

14. Tsang WYW, Chan JKC, Ng CS. Epstein-Barr virus and ReedSternberg-like cells in chronic lymphocytic leukemia. Am J Surg Pathol 1993;17:853-4. 
15. Kuppers R, Rajewsky K, Zhao M, Simons G, Laumann R, Fischer R, et al. Hodgkin's disease: Hodgkin and ReedSternberg cells picked from histological sections show clonal immunoglobulin gene rearrangements and appear to be derived from B cells at various stages of development. Proc Natl Acad Sci U S A 1994;91:10962-6.

16. Kanzler H, Kuppers R, Hansmann ML, Rajewsky K. Hodgkin, and Reed-Sternberg cells in Hodgkin's disease represent the outgrowth of a dominant tumor clone derived from (crippled) germinal center B cells. J Exp Med 1996;184:1495-505.

17. Kanzler H, Kuppers R, Helmes S, Wacker HH, Chott A, Hansmann ML, et al. Hodgkin and Reed-Sternberg-like cells in B-cell chronic lymphocytic leukemia represent the outgrowth of single geminal-center B-cell-derived clones: potential precursors of Hodgkin and Reed-Sternberg cells in Hodgkin's disease. Blood 2000;95:1023-31.

18. Brauninger A, Hansmann ML, Strickler JG, Dummer R, Burg $\mathrm{G}$, Rajewsky $\mathrm{K}$, et al. Identification of common germinalcenter B-cell precursors in two patients with both Hodgkin's disease and non-Hodgkin's lymphoma. N Engl J Med 1999; 340:1239-47.

19. Ohno T, Smir BN, Weisenburger DD, Gascoyne RD, Hinrichs SD, Chan WC. Origin of the Hodgkin/Reed-Sternberg cells in chronic lymphocytic with "Hodgkin's transformation". Blood 1998;91:1757-61.

20. Fend F, Quintanilla-Martinez L, Kumar S, Beaty M, Blum L, Sorbara L, et al. Composite low grade B-cell lymphomas with two immunophenotypically distinct cell populations are true biclonal lymphomas. A molecular analysis using laser capture microdissection. Am J Pathol 1999;154:1857-66.

21. Segal GH, Jorgensen T, Masih AS, Braylan RC. Optimal primer selection for clonality assessment by polymerase chain reaction analysis. Low grade B-cell lymphoproliferative disorders of nonfollicular center cells. Hum Pathol 1994; 25:1269-75.

22. McCarthy KP, Sloane JP, Kabarowski JHS, Matutes E, Wiedemann LM. A simplified method of detection of clonal rearrangements of the T-cell receptor gamma chain gene. Diagn Mol Pathol 1992;1:173-9.

23. Worley J, Lee S, Ma MS, Eisenberg A, Chen HY, Mansfield E. Fluorescence imaging in human identity testing. Biotechniques 1997;23:148-53.
24. Lipa M, Kunynetz R, Pawlowski D, Kerbel G, Haberman H. The occurrence of mycosis fungoides in two patients with preexisting Hodgkin's disease. Arch Dermatol 1982;118:563-7.

25. Müschen M, Rajewski K, Braeuninger A, Baur AS, Oudejans JJ, Roers A, et al. Rare occurrence of classical Hodgkin's disease as a T-cell lymphoma. J Exp Med 2000;191:387-94.

26. Tsang WYW, Chan JKC, Ng CS. The nature of the ReedSternberg-like cells in chronic lymphocytic leukemia. Am J Clin Pathol 1993;99:317-23.

27. Ohshima K, Takeo H, Kikuchi M, Kozuru M, Uike N, Masuda $\mathrm{Y}$, et al. Heterogeneity of Epstein-Barr virus infection in angioimmunoblastic lymphadenopathy type T-cell lymphoma. Histopathology 1994;25:569-79.

28. Wood GS, Bahler DW, Hoppe RT, Warnke RA, Sklar JL, Levy R. Transformation of mycosis fungoides: T-cell receptor beta gene analysis demonstrates a common clonal origin for plaque-type mycosis fungoides and CD30+ large cell lymphoma. J Invest Dermatol 1993;101:296-300.

29. Oshima K, Suzumiya J, Kato A, Tashiro K, Kikuchi M. Clonal HTLV-1-infected CD4+ T-lymphocytes and non-clonal nonHTLV-1-infected giant cells in incipient ATLL with Hodgkinlike histologic features. Int J Cancer 1997;72:592-8.

30. Levi E, Kourea HP, Butmarc J, Kadin ME. Low frequency of Epstein-Barr virus in Reed-Sternberg cells of Hodgkin's disease associated with cutaneous T-cell lymphomas [abstract]. Mod Pathol 1998;11:135A.

31. Zucker-Franklin D, Coutavas EE, Rush MG, Zouzias DC. Detection of human T-lymphotropic virus like particles in cultures of peripheral blood lymphocytes from patients with mycosis fungoides. Proc Natl Acad Sci U S A 1991;88:7630-4.

32. Henghold WB, Purvis SF, Schaffer J, Giam CZ, Wood GS. No evidence of KSHV/HHV-8 in mycosis fungoides or associated disorders. J Invest Dermatol 1997;108:920-2.

33. Bazarbachi A, Soriano V, Pawson R, Vallejo A, Moudgil T, Matutes E, et al. Mycosis fungoides and Sezary syndrome are not associated with HTLV-I infection: an international study. Br J Haematol 1997;98:927-33.

34. Hellman S, Jaffe ES, DeVita VT. Hodgkin's disease. In: DeVita VT, Hellman S, Rosenberg SA, editors. Cancer: principles and practice of oncology. 3rd ed. Philadelphia: JB Lippincott; 1989. pp. 1709-26. 